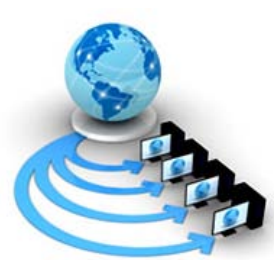

\title{
MULTIMODEL LOCAL TERENARY PATTERNS FOR CONTENT BASED IMAGE RETRIEVAL
}

\author{
Palvi \\ Research Scholar at department of Computer Science \\ Shaheed Bhagat Singh State Technical Campus \\ Ferozepur, Punjab
}

\author{
Er. Sonika Jindal \\ Assistant Professor at department of Computer Science \\ Shaheed Bhagat Singh State Technical Campus \\ Ferozepur, Punjab
}

Abstract: CBIR is the field of digital media that plays important role for extraction of relevant information from media dataset based on various contents. These contents are signals, shapes, and texture and colour compositions. Texture feature provide information about the background of the images and content of the images. LBP has been widely used for texture feature extraction. LBP has major drawback of sensitivity to noise and irregularity at edges. In this paper a novel approach has been utilized for texture feature extraction from the colour images. LTP approach has been used for feature extraction from the colour images so that effective information about the image regions has been computed. Texture features have been computed by decomposing image into various small regions and these features have been concatenated to a single vector. On the basis of proposed model performance we can analyse that proposed system provide better result than existing one.

Keywords: CBIR, LBP, LTP, and Histogram

\section{INTRODUCTION}

1.1 CBIR: Content based image retrieval [1] is based on (automated) matching of the features of the query image with that of image database through some image-image similarity evaluation. Therefore, the images will be indexed according to their own visual content in the light of the underlying (chosen) features like color (distribution of color intensity across image, texture (presence of visual patterns that have properties of homogeneity and do not result from the presence of single color, or intensity), shape (boundaries, or the interiors of objects depicted in the image), or any other visual feature or combination of a set of elementary visual features.

Any CBIR system involves at least four main steps:-Feature extraction and indexing of image database according to the chosen visual features, which form the perceptual feature space, e.g., color, shape, texture or any combination of the above.

- Feature extraction of query image (s).

- Matching the query image to the most similar images in the database according to some image

- image similarity measure this forms the search part of the CBIR

- User interface and feedback which governs the display of the outcomes, their ranking, the type of the user

- Interaction with possibility of refining the search through some automatic or manual preference scheme.

\subsection{Feature Extraction in CBIR:}

Feature extraction is the heart of the content based image retrieval. As we know that raw image data that cannot used straightly in most computer vision tasks. Mainly two reason behind this first of all, the high dimensionality of the image makes it hard to use the whole image. Further reason is a lot of the information embedded in the image is redundant. Therefore instead of using the whole image, only an expressive representation of the most significant information should extract. The process of finding the expressive representation is known as feature extraction and the resulting representation is called the feature vector. Feature extraction can be defined as the act of mapping the image from image space to the feature space. Now days, finding good features that well represent an image is still a difficult task. The most widely recognized technique for contrasting two pictures in substance based picture recovery (ordinarily a sample picture and a picture from the database) is utilizing a picture separation measure. A picture separation measure looks at the similitude of two pictures in different measurements, for example, color, composition, shape, and others. Case in point a separation of 0 implies an accurate match with the question, regarding the measurements that were considered. We can extract the features by using many feature extraction techniques. They are

\subsubsection{Color}

Processing separation measures focused around shade similitude is attained to by registering a color histogram [2] for each one picture that recognizes the extent of pixels inside a picture holding particular values. Examining pictures focused around the colors they contain is a standout amongst the most broadly utilized procedures in light of the fact that it can be finished without respect to picture estimate or orientation. However, inquire about has additionally endeavored to section shade extent by locale and by spatial relationship among a few shade regions.

\subsubsection{Texture}

Surface measures search for visual examples in pictures and how they are spatially characterized. Compositions are spoken to by texels which are then set into various sets, contingent upon what numbers of surfaces are identified in the picture. These sets not just characterize the composition, additionally where in the picture the surface is located. Composition is a troublesome idea to speak to. The distinguishing proof of particular compositions in a picture is accomplished essentially by demonstrating composition as a twodimensional ash level variety [3]. The relative splendor of sets of pixels is figured such that level of differentiation, consistency, coarseness and directionality may be estimated. 
The issue is in distinguishing examples of co-pixel variety and partners them with specific classes of compositions, for example, smooth, or unpleasant.

\subsubsection{Shape}

Shape does not allude to the state of a picture yet to the state of a specific locale that is being searched out. Shapes will regularly be resolved first applying division or edge recognition [4] to a picture. Different routines utilization shape channels to recognize given states of an image. Shape descriptors might likewise need to be invariant to interpretation, pivot, and scale.

\section{REVIEW OF LITERATURE}

Shiv Ram Dubey et al [5] "Multichannel Decoded Local Binary Patterns for Content-Based Image Retrieval”, Local binary pattern (LBP) is widely adopted for efficient image feature description and simplicity. To describe the color images, it is required to combine the LBPs from each channel of the image. The traditional way of binary combination is to simply concatenate the LBPs from each channel, but it increases the dimensionality of the pattern. In order to cope with this problem, this paper proposes a novel method for image description with multichannel decoded LBPs. We introduce adder- and decoder-based two schemas for the combination of the LBPs from more than one channel. Image retrieval experiments are performed to observe the effectiveness of the proposed approaches and compared with the existing ways of multichannel techniques.

Xavier, L. et al [6] "Content based image retrieval using textural features based on pyramid-structure wavelet transform", Picture Retrieval framework is a viable and effective instrument for overseeing extensive picture databases. A substance based picture recovery framework permits the client to present a question picture to recover pictures put away in the database as per their closeness to the inquiry picture. In this paper substance based picture recovery system was utilized as conclusion support as a part of therapeutic fields. The fundamental destination of this paper was to assess the recovery framework focused around Texture characteristics. The surface peculiarities were separated by utilizing pyramidal wavelet change. The real preference of such a methodology was, to the point that minimal human mediation was needed. The technique was assessed on Diabetic Retinopathy Database (DRD). Here the exactness rate acquired was around $60 \%$ for DRD pictures.

Choudhary, R. Et al [7] "An integrated approach to Content Based Image Retrieval”, Substance based picture recovery, in the last few years has gotten a wide consideration. Substance Based Image Retrieval (CBIR) fundamentally is a method to perform recovery of the pictures from a huge database which is like picture given as question. CBIR is closer to human semantics, in the connection of picture recovery process. CBIR method has its application in distinctive spaces, for example, wrongdoing aversion, therapeutic pictures, climate determining, observation, authentic examination and remote sensing Here substance alludes to the visual data of pictures, for example, composition, shape and shade. Substance of picture is wealthier in data for a proficient recovery in correlation to content based picture recovery. In this paper, author proposed a substance based picture recovery coordinated strategy which removes both the color and composition characteristic.
Dharani, T et al [8] "A survey on content based image retrieval”, Writing overview is most essential for understanding and increasing significantly more learning about particular territory of a subject. In this paper an overview on substance based picture recovery displayed. Substance Based Image Retrieval (CBIR) is a method which utilizes visual peculiarities of picture, for example, color, shape, composition, and so forth to hunt client obliged picture from expansive picture database as per client's appeals as a question picture. Author consider Content Based Image Retrieval viz. named and unlabeled pictures for breaking down effective picture for diverse picture recovery process viz. DEM, SVM, RF, and so on. To deciding the proficient imaging for Content Based Image Retrieval, author execution writing survey by utilizing standards of Content Based Image Retrieval based unlabeled pictures. Furthermore provide for a few suggestions for enhance the CBIR framework utilizing unlabeled pictures.

Sreedevi, S. et al [9] "Content based image retrieval based on Database revision" Pictures are the least complex and most ideal method for representation of thoughts. The criticalness of pictures has been impressively expanded by the site pages. Consequently effective picture recovery frameworks are crucial. Substance based picture recovery (CBIR) frameworks are the most recent range of exploration. In this paper, a canny picture recovery framework focused around a novel technique called database update (DR) was proposed. Picture characteristic extraction regarding color, surface and shape was utilized to recover pictures from the database. The after effect of gimmick likeness examination of the inquiry picture with database pictures revises the database. The framework was made intuitive for the clients to recognize the pictures that were most fulfilled to the need. The client fulfilled pictures were broken down and the database was reexamined to make the framework adroit. Test results and correlations are exhibited to show the attainability of the proposed technique.

Rai, H.G.N. et al [10] "Hybrid feature to encode shape and texture for Content Based Image Retrieval” In this paper, author was proposed a methodology for speaking to both shape and composition data in a picture utilizing a solitary half breed gimmick descriptor for Content Based Image Retrieval. Towards this, we process the inclination size of the data picture before inferring gimmicks. Characteristic extraction is then performed utilizing the reactions from a bank of Gabor channels. Here, author abused the way that shape compares to the high spatial recurrence content in the picture while common composition data overwhelmingly exists in low to mid-range frequencies. This methodology helps in better restriction of trademark surface and shape, because of spread of vitality towards high frequencies in ghastly area. Minute invariants were extricated from Gabor channel reactions which yield preferable recovery execution over ordinary factual peculiarities.

\section{METHODOLOGY}

Content based image retrieval is used for extraction of similar images from large database using query image. Query image has been provided as input to the system and similar image to the query image has been returned by the system. In this process various features have been used for retrieval system. In proposed method texture features have been used for extraction of similar images to the dataset images. In this process local ternary pattern approach has been utilized that computes texture features from the images. Local ternary 
patterns (LTP) is an approach that is based on center pixel value and threshold values so that features from the boundary region, uniform regions and non-uniform regions can be evaluated. In this process a window of 3 by 3 has been moved on the image pixel regions and this window has been used for computation of local ternary patterns that has been used for computation of feature values. These local ternary patterns have been sub-divided into two different classes that are upper ternary codes and lower ternary codes. In this process of the system 3 by 3 window has been known as mask that computes upper ternary codes and lower ternary codes. The illustration of whole process has been described in section 3.1.

\subsection{LTP Feature Extraction}

In this process of feature extortion a window of 3 by 3 has been selected from the image that has been represented below.

\begin{tabular}{|c|c|c|}
\hline 112 & 125 & 136 \\
\hline 58 & 120 & 198 \\
\hline 160 & 95 & 100 \\
\hline
\end{tabular}

This table represents a window of 3 by 3 from image pixel. In this process image has been divided into two values that are center pixel value and neighbor pixel value. $I_{c}$ and $I_{n}$ represents center pixel value and neighbor pixel value respectively. To compute the ternary codes $\mathrm{S}(\mathrm{x})$ has been computed by using $\mathrm{I}_{\mathrm{c}}$ and $I_{n}$.

$$
x_{n}=\sum_{n=0}^{7}\left(I_{c}-I_{n}\right)
$$

In this proposed system a threshold value $t$ that is 10 has been used for computation of ternary codes.

$$
S(x)=\left\{\begin{array}{c}
1 x \geq t \\
0|x|>t \\
-1 x \leq-t
\end{array}\right.
$$

On the basis of equation one and two above defined window has been converted to different codes that are represented below.

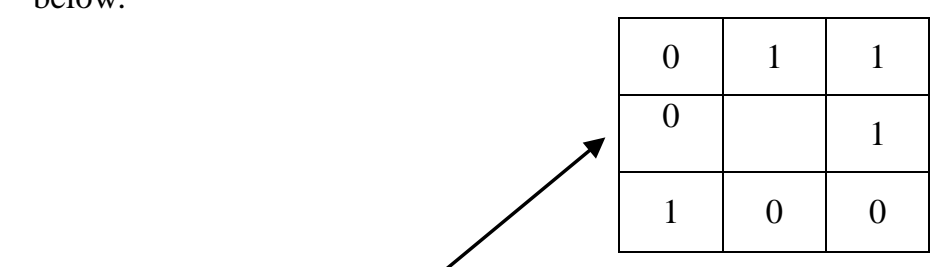

\begin{tabular}{|c|c|c|}
\hline 0 & 1 & 1 \\
\hline-1 & & 1 \\
\hline 1 & -1 & -1 \\
Lower codes \\
$\qquad$\begin{tabular}{|l|l|l|l|}
\hline 0 & 0 & 0 \\
\hline 1 & & 0 \\
\hline 0 & 1 & 1 \\
\hline
\end{tabular}
\end{tabular}

After this process of conversion of upper and lower ternary codes these codes have been converted to decimal values and these values have been stored as feature vectors. From above example upper code is 01110010 that have decimal value 114 and lower ternary code that is represented as 00001101 that have decimal value 13 . These values have been taken as feature values from the 3 by 3 window. Features have been computed for all the regions of the image so that feature vector can be developed.

Texture features have been computed for the single color space or gray scale images. In this process for feature extraction of the color images image has been subdivided into RGB color space model. After decomposition into RGB color space model LTP features for all color spaces has been computed and stored in single vector so that a feature vector can be developed for colored image.

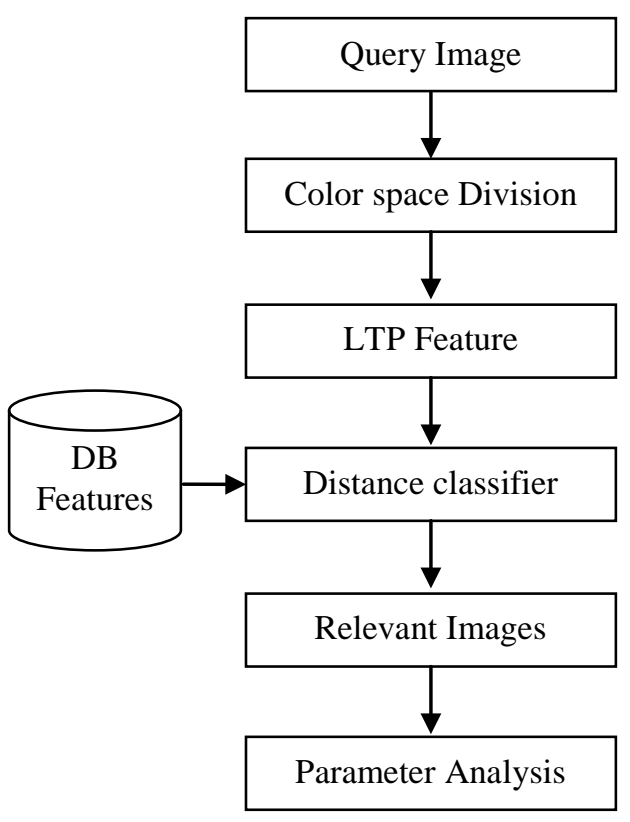

Fig 3.1 Flow of CBIR Model

This figure represents step wise flow of the proposed system that has been used for extraction of relevant images from the dataset images. In this process numbers of relevant images have been retrieved from the dataset that are relevant to dataset images.

Texture feature plays a vital role in retrieval process. These features provide information about the content available in the images so that images can be retrieved based on contents.

\section{RESULTS}

In the proposed work relevant images from the dataset has been extorted using texture features that are computed based on LTP.

Corel 1-K dataset has been used in the proposed model that contains images of 10 different categories. These categories are African people, beach, buses, buildings, dinosaurs, elephants, horses, flower, mountains, and food. In the dataset each category contains 100 images. Feature for entire dataset has been computed and used for extraction of relevant images to the content of query image.

On the basis of extraction of relevant images different parameters have been analyzed for proposed system. These parameters are precision, recall and computation time.

- Precision: it is the fraction of the total relevant images to the total retrieved images that are extracted from the dataset. 


\section{$P=\frac{\text { Total relevent images out of retrived images }}{\text { Total number of images retrived }}$}

- Recall: it is the fraction of the relevant image to the total no of relevant images available in the dataset.

$$
R=\frac{\text { Total relevant images out of retrived images }}{\text { Total number of relevant images in database }}
$$

- Computation Time: it is measure to check the time complexity of the algorithm. This computes total time taken by the algorithm for feature extraction.

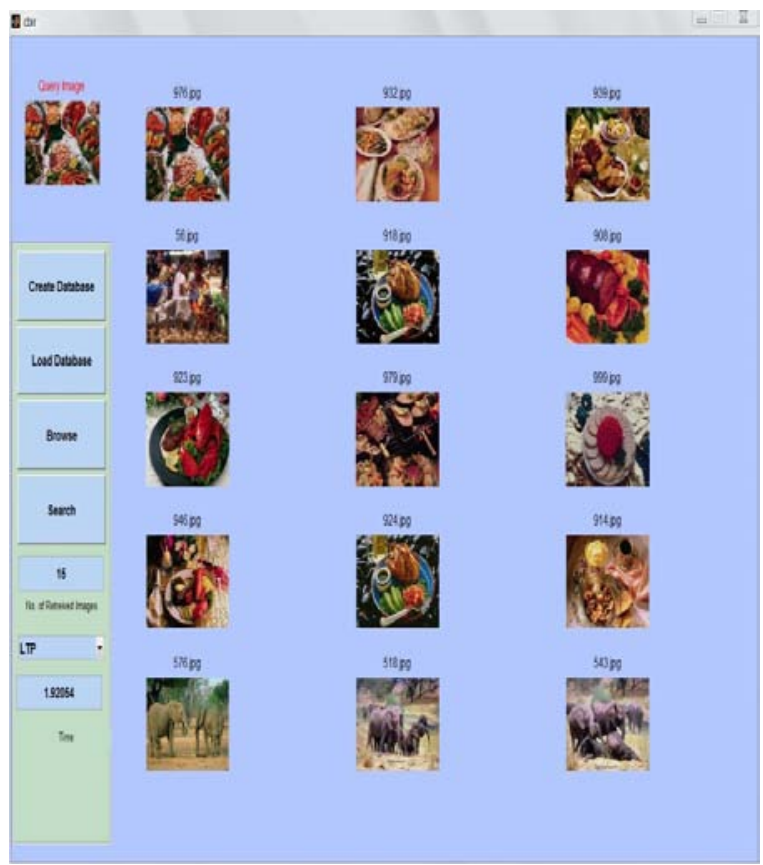

Fig 4.1 Retrieved images based on food category

This figure represents images that have been retrieved on the basis of food image that has been given to the system as input image. On the basis of input image features of image has been computed using LTP approach. This approach provides information about the texture alignment of the food particles. On the basis of these information relevant images from the dataset has been extorted.

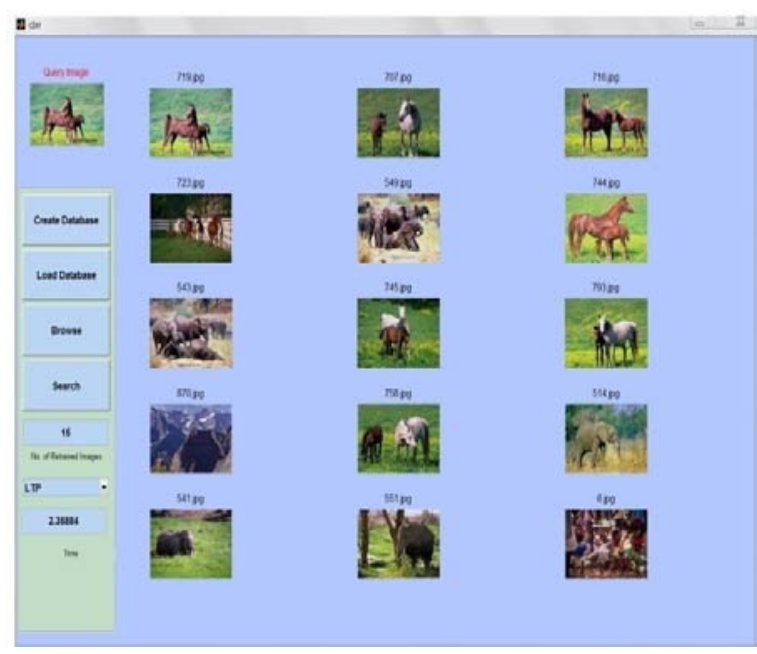

Fig 4.2 Retrieved images based on horse category

This figure represents images that have been retrieved on the basis of horse image that has been given to the system as input image. On the basis of input image features of color compositions, texture and shape has been computed that provide information about relevant images.

In the proposed work 15 images has been retrieved based on query image. On the basis of these 15 images performance evaluation parameters have been evaluated for proposed system.

Table 4.1 Parameter Analysis of Proposed Approach

\begin{tabular}{|c|c|c|c|}
\hline Database & Precision & Recall & $\begin{array}{c}\text { Computation } \\
\text { time }\end{array}$ \\
\hline Africa & 0.93 & 0.14 & 0.93 \\
\hline Beach & 0.53 & 0.08 & 0.53 \\
\hline Building & 0.93 & 0.14 & 0.93 \\
\hline Bus & 1 & 0.15 & 1 \\
\hline Dinosaur & 0.93 & 0.14 & 0.93 \\
\hline Elephant & 0.67 & 0.1 & 0.67 \\
\hline Flower & 0.93 & 0.14 & 0.93 \\
\hline Food & 0.93 & 0.14 & 0.93 \\
\hline Horses & 0.87 & 0.13 & 0.87 \\
\hline Mountain & 0.6 & 0.09 & 0.6 \\
\hline Average & 0.832 & 0.44365 & 0.832 \\
\hline
\end{tabular}

This table represents performance evaluation parameters that have been computed for proposed work on different category basis and for over all image dataset on the basis of average values.

\section{Precision Recall Curve}



Fig 4.3 Comparative Analysis based on precision recall curve This figure represents comparison between proposed approach and existing approach in graphical representation. This graph represents precision and recall curve for both approaches. On the basis of these curve comparison can be made between proposed and existing approach. 


\section{CONCLUSION \& FUTURE SCOPE}

Texture Features are widely used in multimedia applications. Texture features plays an important role in content based image retrieval system. In this process of retrieval texture descriptors have been used so that effective features can be evaluated. Various approaches that are based on energy, contrast and correlation had been proposed for texture feature analysis. These approaches does not provide correct information due to sensitive to noise so in this paper an approach has been adopted for content based image retrieval system that is based on local ternary pattern. Proposed approach provides better precision and recall than existing approaches.

\section{REFRENCES}

[1] Jiyi Li "Context-based image re-ranking for content-based image retrieval" in International Conference 2011 on Computational Intelligence for Multimedia, Signal and Vision Processing (CIMSIVP),pp. 39 - 46.

[2] Zhongmiao Xiao "Block-based long-term content-based image retrieval uses multiple features" in IEEE International Conference on Multimedia and Expo (ICME), pp. 1 - 6.

[3] Bo Li "A UIM/ICM based approach to content-based image retrieval” in IEEE International Conference 2013 on Multimedia and Expo (ICME), pp. 1 - 6, IEEE,2013.
[4] EsmatRashedi, Hossein Nezamabadi-pour "Improving the Precision of CBIR Systems by Feature Selection UsingBinary Gravitational Search Algorithm”, in 16th CSI International Symposium on Artificial Intelligence and Signal Processing, IEEE, 2012, ISBN No. 978-1-4673-1479-4, pp. 39-42.

[5] Shiv Ram Dubey, Satish Kumar Singh, Rajat Kumar Singh, "Multichannel Decoded Local Binary Patterns for ContentBased Image Retrieval”, IEEE transactions on image processing, 2016,pp. 4018-4025

[6] Xavier, L. , Thusnavis, B.M.I. , Newton, D.R.W. “Content based image retrieval using textural features based on pyramidstructure wavelet transform", in 3rd International Conference on Electronics Computer Technology (ICECT), 2011, vol 4, ISBN No. 978-1-4244-8679-3, pp. 79-83.

[7] Choudhary, R., Raina, N., Chaudhary, N. ,Chauhan, R “An integrated approach to Content Based Image Retrieval”, in International Conference 2014 on Advances in Computing, Communications and Informatics, IEEE, ISBN No. 978-1-47993078-4, pp. $2404-2410$.

[8] Dharani, T, Aroquiaraj, I.L. “A survey on content based image retrieval" in International Conference 2013 on Pattern Recognition, Informatics and Mobile Engineering (PRIME), ISBN No. 978-1-4673-5843-9, pp. 485 - 490.

[9] Sreedevi, S. "Content based image retrieval based on Database revision”, in Machine Vision and Image Processing (MVIP), 2012 International Conference, pp. 29-32, IEEE, 2012.

[10] Rai, H.G.N., "Hybrid feature to encode shape and texture for Content Based Image Retrieval” in International Conference 2011 onImage Information Processing (ICIIP), pp. 1-6. 